\title{
Management of Chronic Hepatitis C Virus Infection: Current Perspectives
}

\author{
Murtaza Mustafa ${ }^{1,}{ }^{\text {Jayaram Menon }}{ }^{2}$,EL.Illzam ${ }^{3}$, AM.Sharifa ${ }^{4}$, \\ RK.Muniandy ${ }^{5}$,A.Nornazirah ${ }^{6}$ \\ ${ }^{1,5,6}{ }^{F a c u l t y}$ of Medicine and Health Sciences, University Malaysia, Sabah,KotaKinabalu,Sabah,Malaysia \\ ${ }^{2}$ Department of Gastroenterology, Hospital queen Elizabeth, KotaKinabalu,Sabah,Malaysia \\ ${ }^{3}$ Clinic Family PlanningAssociation,Kota Kinabalu,Sabah,Malaysia \\ ${ }^{4}$ Quality Unit Hospital Queen Elizabeth, Kota KInabalu,Sabah,Malaysia
}

\begin{abstract}
Worldwide over 300,000 deaths due to liver cancer and cirrhosis occurred in 2013 due to hepatitis C. In the United States about $2 \%$ of population has chronic hepatitis C. In 2014 it was the single greatest cause of death in the United States. The number of deaths due to hepatitis $C$ has overtaken HIV/AIDS as a cause of death in USA. Hepatitis $C$ virus (HCV) infection is also prevalent in Africa, Central and East Asia, with highest rates in Egypt. Transmission of $\mathrm{HCV}$ in the developed world is intravenous drug use, while in the developing countries the main method of transmission is blood transfusion and unsafe procedures. Once the infecting virions reaches the susceptible cells that are permissive replication.HCV RNA can be detected in blood(including serum and plasma),tears, seminal fluid, ascetic fluid, and cerebrospinal fluid. HCV infection leads to hepatic inflammation, steatosis, cirrhosis, and greater risk of hepatocellular carcinoma. Clinical manifestations include acute infection, chronic infection, non-hepatic complications, and occult infection.Diagnosis including HCV antibody enzyme immunoassay (ELISA), recombinant,andquantitative HCV RNA PCR.Blood tests alleviate the need for liver biopsy.Sofosbuvir with ribavirin and interferon is frequently used drugs. Virus usually (80-90\% of cases) recurs in liver transplant patients. Alternative therapies e.g. Milkthistle, ginseng and colloidal gold have no effect on the virus at all. Successful treatment decreases the future risk of hepatocellular carcinoma by $75 \%$. Prevetion include screening of blood donors and adhering to universal precautions within the healthcare system.
\end{abstract}

Keywords: Chronic hepatitis C, Transmission, Management, Prognosis

\section{Introduction}

Hepatitis $\mathrm{C}$ is an infectious disease caused by the hepatitis $\mathrm{C}$ virus(HCV) that primarily affects the liver[].It is one of five known hepatitis viruses:A,B,C,D, and E[2].An estimated 130 to 200 million people or $3 \%$ of the world's population are living with chronic hepatitis[3].About 3-4 million people are infected per year, and more than 350,000 people die yearly from hepatitis C related diseases[3].During 2010 it is estimated that 16,000 people died from acute infections while 196,000 deaths occurred from liver cancer secondary to the infection[4].It occurs most commonly in Africa and Central and East Asia[5].About 343,000 deaths due to liver cancer and 358,000 due to cirrhosis occurred in 2013 due to hepatitis C[6].In the United States, about 2\% of people have chronic hepatitis C[7].The number of deaths from hepatitis $\mathrm{C}$ has increased to 15,800 in 2008, having overtaken HIV/AIDS as a cause of death in the USA in 2007[8,9].In 2014 it was the single greatest cause of infectious death in the United States[10].In Europe the percentage of people with chronic infection has been estimated to be between 0.13 and 3.26[11].Countries with particularly high rates include Egypt(22\%),Pakistan(4.8\%) and China 3.2\%[3].Transmission of HCV is primarily by blood -to-blood contact with intravenous drug use, poorly sterilized equipment, needle stick injuries in the healthcare, and transfusion[12].During the initial infection people have mild or no symptoms.Occasionally a fever, dark urine,abdominal pain, and yellow tinged skin. Virus persists in the liver in about $75 \%$ to $85 \%$ of those initially infected, and later often leads to liver disease and occasionally cirrhosis[12].Diagnosis by blood testing for virus antibodies or RNA. Testing is recommended in all people who are at risk[12].Chronic infection can be cured about $90 \%$ of the time with treatments that include the medications sofosbuvir or simeprevir [12].People with cirrhosis or liver cancer may require liver transplantation, though the virus usually recurs after transplantation[13].The reviews the current literature on management of hepatitis $\mathrm{C}$ virus infections.

\section{Discovery ofHepatitis C Virus}

When serologic tests for hepatitis $\mathrm{A}(\mathrm{HAV})$,and hepatitis $\mathrm{B}$ virus(HBV) were developed during the 1970s, it became evident that most cases of transfusion associated hepatitis must be caused by yet another agent, leading to the term non-A, non-B hepatitis(NANB)[14].In 1987, Michael Houghton and associates at Chiron 
Corporation, collaborating withDr DW Bradley at the Centers for Disease Control and Prevention(CDC), used a novel molecular cloning approach to identify the unknown organism and developed a diagnostic test [15].In 1988, Alter confirmed the virus by verifying its presence in a panel of NANBH specimens. In April 1989, the discovery of HCV was published in two articles in the journal Science[16].The discovery led to significant improvements in diagnosis and improved antiviral treatment [15],In 2000,Drs,Alter and Houghton were honored with the Lasker Award for Clinical Medicine Research for "pioneering work leading to discovery of the virus that causes hepatitis $\mathrm{C}$ and the development of screening methods that reduced the risk of blood transfusionassociated hepatitis in the U.S.from $30 \%$ in 1970 to virtually zero in 2000"[17].

\section{Hepatitis C Virus}

Hepatitis C virus (HCV) is a small, enveloped, single-stranded, positive-sense RNA virus[13].It is a member of the Hepacivirus genus in the family Flaviridae[18,wp,20].There are seven major genotypes of $\mathrm{HCV}$, which are known as genotypes one to seven[19].The genotypes are divided into several subtypes with the number of subtypes depending on the genotype. In the United States, about $70 \%$ of cases are by genotype $1,20 \%$ by genotype two and $1 \%$ by each other genotypes[7].Genotype 1 is also the most common in South America and Europe[13].The half-life of the virus in the serum is around 3 hours and may be as short as 45 minutes[20].In addition to replicating in the liver the virus can multiply in lymphocytes[21].

\section{HCV and genetic variation}

The high level of vision turnover, the absence of proofreading by theNS5B RNA polymerase, and the "tolerance" of many genomic regions for multiple nucleotides results in the relatively rapid accumulation of viral mutations. Multiple HCV variants can be recovered from the plasma and liver of an infected individual at time.AS a results, like many RNA viruses,HCV exists in each infected person as a quasi-species, or "swarm" of closely related but distinct genetic sequences[22].For example, up to $85 \%$ of cDNA clones obtained from viral RNAs in the blood of a recently infected individual may present unique genetic variants[23].During RNA, replication, mutations most likely occur in a nearly, random fashion throughout the genome, whereas fixation of a substitution within the quasi-species population depends on how that substitution influences viral "fitness" as related to its effect on functional protein/RNA structures, the capacity of the virus for replication, and the host viral interaction[18].

\section{Transmission ofHCV}

HCV transmission requires that infecting virions contact susceptible cells that are permissive for replication.HCV RNA can be detected in blood (including serum and plasma),saliva, tears, seminal fluid,asciticfluid and cerebrospinal fluid[24].HCV- RNA-containing blood is infectious when inoculated intravenous(e.g., by transfusion).In addition a chimpanzee has been infected by intravenous inoculation of saliva[25].However, there is very little information available regarding the potential infectivity of HCV-RNA containing body fluids.Furthermore, it is not clear whether cells other than hepatocytes can be infected (and thus whether infection requires direct percutaneous inoculation into the blood stream)[18].

The primary route of transmission in the developed world is intravenous drug use(IDU), while in the developing world the main methods are blood transfusion and unsafe medical procedures[26].The cause of transmission is unknown in $20 \%$ of cases[27],however, many of these are believed to be accounted for by IDU[28].

\section{Intravenous drug use}

IDU is a major risk factor for hepatitis $\mathrm{C}$ in many parts of the world [29].Off 77 countries reviewed 25(including the United States) were found to have prevalence of hepatitis $\mathrm{C}$ in the intravenous drug user population of between $60 \%$ to $80 \%$ [29,].Twelve countries had rates greater than $80 \%$ [30].It is believed that ten million intravenous drug users are infected with hepatitis China(1.6 million), the United States(1.5 million), and Russia(1.3 million) have the highest absolute totals[30].Occurrence of hepatitis $\mathrm{C}$ among prison inmates in the United States is 10 to 20 times that of the occurrence observed in the general population, this has attributed to high-risk behavior in prisons such as IDU and tattooing with nonsterile equipment[31].Shared intranasal drug use may also be a risk factor[32].

\section{Nosocomial infection}

Blood transfusion, transfusion of blood products, or organ transplants without HCV screening carry significant risks of infection [7].The United States instituted universal screening in 1992, and Canada instituted universal screening in 1990[33,34].This decreased the risk from on in 200 units[33],to between on in 10,000 to $10,000,000$ per unit of blood[28,].This low risk remains as there is a period about 11-70 days between the 
potential blood donor's acquiring hepatitis $\mathrm{C}$ and the blood's testing positive depending on the method[27].Some countries do not screen for hepatitis $\mathrm{C}$ due to the cost [35].

Those who have experienced a needle stick injury from someone who was HCV positive have about a1.8\% chance of subsequently contracting the disease themselves[7].The risk is greater if the needle in question is hollow and puncture wound is deep[35].there is risk from mucosal exposures to blood, risk is low, and there is no risk if blood exposure occurs on intact skin[35].Hospital equipment has also been documented as a method of transmission of hepatitis Including reuse of needles and syringes, multiple-use medication vials, infusionbags, and improperly sterilized surgical equipment,among others[35].Limitations in the implementation and enforcement of stringent standard precautions in public and private medical and dental facilities are known to be the primary cause of the spread of HCV in Egypt, the country with highest rate of infection in the world [36].

\section{Sexual transmission}

Whether hepatitis C can be transmitted through sexual activity is controversial [37]..While there is an association between high-risk sexual activity and hepatitis $\mathrm{C}$ and multiple sexual partners are a risk factor for hepatitis $\mathrm{C}$, there is no conclusive evidence that hepatitis $\mathrm{C}$ can be transmitted by sexual activity, since people who report transmission with sex as their only risk factor may actually have used drugs but denied[7].The majority of evidence supports there being no risk for heterosexual couples with only one sexual partner[37].Sexual practices that involve higher levels of trauma to the anogenitalmucosa, such as anal penetrative sex or that occur when there is a concurrent sexually transmitted infection including HIV or genital ulceration, do present a risk[37]The United States Department of Veterans Affairs recommend condom use to prevent hepatitis $\mathrm{C}$ transmission in those with multiple partners, but not those in relationships that involve only a single partner[38].

\section{Common use items}

Personal use-shared items such as razors, toothbrushes, and manicuring of pedicuring equipment can be contaminated with blood. Sharing such items can potentially lead to exposure to HCV[39].Appropriate caution should be taken regarding and medical condition that results in bleeding, such as cuts and sores[40].HVC is not spread through casual contact, such as hugging, kissing or sharing eating or cooking utensils [40].Neither it is transmitted through food or water[41].

\section{Body Tattooing}

Tattooing is associated with two to threefold increased risk of hepatitis C[42].This can be due to either improperly sterilized equipment or contamination of the dyes being used[42].Tattoos or piercings performed either before the mid-1980s,"underground" or nonprofessionally are particular concern, since sterile techniques in such settings may be lacking. The risk also appears to be greater for larger tattoos [42].It is estimated that nearly half the prison inmates share unsterilized tattooing equipment[42]..It is rare for tattoos in a licensed facility to be directly associated with HCV infection [43].

\section{Maternal transmission}

Maternal or mother to child transmission of hepatitis $\mathrm{C}$ is less than $10 \%$ of pregnancies[44].There no measures that alter this risk[44].It is not clear when transmission occurs during pregnancy, but it may occur both during gestation and delivery[27].A long labor is associated with a greater risk of transmission[35].There is no evidence that breast feeding spreads HCV ,however, to be cautions, an infected mother is advised to avoid breastfeeding if her nipples are cracked and bleeding[45], or if viral loads are high[27].

\section{Transmission Cofactors[18].}

Cofactors for HCV transmission chiefly relate to the probability of virus reaching the recipient bloodstream. The donor HCV RNA level matters, especially at the extremes. In a review of 2022 parenteral, sexual, and perinatal HCV exposures, HCV was transmitted only by individuals with detectable viremia [46].Moreover, non-parenteral(e.g., perinatal) transmission of HCV is very rare when the level of viremia is very low[47].Some studies not all studies indicate the infection with HIV may be an important cofactor for both sexual and maternal-infant transmission[48], possibly because HIV infection is also associated with higher HCV RNA levels[49].

\section{V.Pathogenesis}

In experimentally infected chimpanzee and in humans HCV RNA can be detected in plasma within days of exposure, often 1 to 4 weeks before liver enzyme levels rise [50].Viremia peaks in the first 8 to 12 weeks of infection, then drops to lower levels and persists[51].In some instances, plasma HCV RNA becomes undetectable in the first few months and remains undetectable indefinitely(viral clearance);in other instances, 
viremia is inconsistently detected early and stable pattern of recovery or persistence is not evident for more than six months[51].Some instances of intermittent viremia may reflect reinfection, which has been observed in active injection drug users[52].

Within months of infection, antibodies are detectable in blood to multiple recombinant antigens that correspond to structural and non-structural genes [53]. Emergence of HCV-specific antibodies does not correlate temporally with viral recovery.Indeed, although virtually all immunocompetent persons develop antibody responses to someHCV antigens, most infection persist. Viral recovery also has described in persons with congenital agammaglobulinemia[54]. Viral recovery has been associated with a vigorous, blood cellular immune response[55].It appears that in persons with persistent infection, $\mathrm{CD} 4^{+}$lymphocyte responses are more difficult to detect in peripheral blood than $\mathrm{CD}^{+}$lymphocyte responses[56].There is surprisingly little known about why some cellular immune responses are broad and vigorous and others ineffective. Coinfection with HIV or schistosomiasis has been associated with viral persistence, which corresponds with diminished $\mathrm{CD}^{+}$ lymphocyte response [57].

Although HCV infection leads to hepatic inflammation and steatosis the major pathologic consequence of persistence $\mathrm{HCV}$ infection is the development of hepatic fibrosis, which may progress to life threatening cirrhosis and greatly risk of hepatocellular carcinoma. These long- term complications generally occur more than 20 years after the onset of infection, though more rapid progression has been reported [58].

\section{Clinical Manifestations}

\section{Acute infection}

Hepatitis C infection causes acute symptoms in $15 \%$ of cases[59].Symptoms are generally mild and vague, including a decreased appetite, fatigue, nausea, muscle or joint pain, and weight loss[7], and rarely acute liver failure result[60]. Most cases of cute infection is not associated with jaundice[61]. The infection can be resolved spontaneously in 10-50\% of cases, which occurs more frequently in individuals who are young and female[61].HCV RNA can be detected in blood within days of exposure and is followed by elevations in serum levels of liver specific enzyme and alanine aminotransferase(ALT), aspartate aminotransferase(AST) and in some cases bilirubin [62].

\section{Chronic infection}

About $80 \%$ of those exposed to the virus develop chronic infection [30].This is defined as the presence of detectable viral replication for at least for six months. Most experience minimal or no symptoms during the initial few decades of the infection [63].Chronic hepatitis may be associated with fatigue[18], and mild cognitive problem [64].Chronic infection after several years may cause cirrhosis or liver cancer[13].The liver enzymes are normal in 7-53\%. The late relapses after apparent cure have been reported but these can be difficult to distinguish from reinfection[65].

Fatty changes to the liver occur in about half of those infected and are usually present before cirrhosis develops [66].Usually (80\% of the time) this change affects less than a third of the liver[66].Worldwide hepatitis $\mathrm{C}$ is the cause of $27 \%$ of cirrhosis cases and $25 \%$ hepatocellular carcinoma[35]. About 10 t0 $30 \%$ of those infected develop cirrhosis over 30 years[13,7]..Cirrhosis is more common in those also infected with hepatitis B, Schistosoma, orHIV,inalcoholics and those of male gender[7].In those with hepatitis C,excess alcohol increases the risk of developing cirrhosis 100 fold[67].Those who develop cirrhosis have a 20 fold greater risk of hepatocellular carcinoma. This transformation occurs at a rate of 1-3\% per year [13,7].Being infected with hepatitis $\mathrm{B}$ in addition to hepatitis $\mathrm{C}$ increases this risk further[68].Liver cirrhosis may lead to portal hypertension, ascites(accumulation of fluid in the abdomen) easy bruising or bleeding, varicose (enlarged veins, especially in the stomach and esophagus),jaundice, and a syndrome of cognitive impairment known as encephalopathy[69]. Ascites occurs at some stage in more than half of those who have chronic infection[70].

\section{Non-hepatic complications}

The common problem due to hepatitis $\mathrm{C}$ but not involving the liver is mixed cryoglobulinemia (usually the type 11 form)-an inflammation of small and medium-sized blood vessels [71].Hepatitis $\mathrm{C}$ is also associated with autoimmune disorder Sjogrensyndrome,a low platelet count, lichen planus porphyria cutanea tarda,neurolytic acral erythema, insulin resistance, diabetes mellitus, diabetic nephropathy, autoimmune thyroiditis,and B-cell lymphoproliferative disorders[72],20-30\% of people infected have rheumatoid factor-a type of antibody [73].Possible association include Hyde's prurigonodularis [74], and membranoproliferative glomerulonephritis[65].Cardiomyopathy with associated with abnormal heart rhythms has also been reported[75].A variety of central nervous system disorders has been reported. Chronic infection seems to be associated with an increased risk of pancreatic cancer [76,77]. 


\section{HCV negative or occult infection}

Persons who have been infected with hepatitis $\mathrm{C}$ may appear to clear the virus but remain infected. The virus is not detectable with conventional testing but can be found with ultra-sensitive tests[78,79].The virus can be detected by antibody test for virus core protein and the viral genome[80].It may be found in people with antihepatitis C-antibodies but with normal levels of liver enzymes 'in antibody- negative people with ongoing elevated liver enzymes of unknown cause, in healthy populations without evidence of liver disease, and groups at risk for HCV infection including those on hemodialysis or family members of people with occult HCV.The clinical relevance of this form of infection is under investigation [81].The rate of occult infection in those apparently cured is controversial but appears to be low[66].

\section{$\mathrm{HCV}$ in children and pregnancy}

Worldwide the prevalence of hepatitis $\mathrm{C}$ virus infection in pregnant women and children has been estimated to $1-8 \%$ and $0.05-5 \%$ respectively [84].Vertical transmission has been estimated to be $3-5 \%$ and there is a high rate of spontaneous clearance (25-50\%) in the children. Higher rates have been reported for both vertical transmission(18\%,6-36\%) and 41\%)[83], and prevalence in children(15\%)[84].Factors associated with an increased rate of infection include membrane rupture of longer than 6 hours before delivery and procedures exposing the infant to maternal blood[85]. Higher $\mathrm{HCV}$ infection has been reported in immunocompromised and those with human immunodeficiency virus infection, recipients of organ transplants, and those with hypogammaglobunemia. Infection in these people is associated with an unusually rapid progression to cirrhosis [86].

\section{Diagnosis}

There are variety of diagnostic tests for hepatitis, including HCV antibody enzyme immunoassay or ELISA, recombinant, and quantitative HCV RNA polymerase chain reaction (PCR)[7]HCV RNA can be detected by PCR typically one to two weeks after infection, while antibodies can take substantially longer to form and thus be detected[69t].Chronic hepatitis $\mathrm{C}$ is defined as infection with the hepatitis virus persisting for more than six months based on the presence of its RNA[63].Chronic infections are typically asymptomatic during first few decades, and thus are most commonly discovered following the investigation of elevated liver enzymes levels or during screening of high risk individuals. Testing is not able to distinguish between acute and chronic infections [63,35].Diagnosis in the infant is difficult as maternal antibodies may persist for up to 18 moths[87].

\section{Serological tests}

Hepatitis $\mathrm{C}$ testing typically begins with blood testing to detect the presence of antibodies to the HCV, using an enzyme immunoassay[7]If test is positive, a confirmatory test is then performed to verify the immunoassay and to determine the viral load[7].A recombinant immunoblot assay is used to verify the immunoassay and the viral load is determined by an HCV RNA polymerase chain reaction[7].If there is no RNA and the immunoblot is positive, it means that person tested had previous infection but cleared it either with treatment or spontaneously; if the immunoblot is negative, it means that immunoassay was wrong[7].It takes about 6-8 weeks following infection before the immunoassay will test positive[18].Liver enzymes are variable during initial part of infection[63], and on average begin to rise at seven weeks after infection[18].The elevation of liver enzymes does not closely follow disease severity[18].

\section{Biopsy and Screening}

Liver biopsies are used to determine the degree of liver damage present, however, there are risks from the procedure[13].The typical changes seen are lymphocytes within the parenchyma, lymphoid follicles in portal triad, and changes to the bile ducts[13].There are number of blood tests available that try to determine the degree of hepatic fibrosis and alleviate the need for biopsy[13].Screening for $\mathrm{HCV}$ is recommended for those at high risk, which includes injecting drug users, those who have received blood transfusion before 1992[88],those have been in jail, those on long term hemodialysis[89], and those with tattoos[42].Screening also recommended in those with elevated liver enzymes, as this frequently the only sign of chronic hepatitis[90].Routine screening is not recommended in the united States[7].

\section{Management}

$\mathrm{HCV}$ induces chronic infection in $50-80 \%$ of infected persons. Approximately $40-80 \%$ of these clear with treatment[91].In rare cases infection can clear without treatment[28].Those with chronic hepatitis $\mathrm{C}$ are advised to avoid alcohol and medications toxic to liver[7].Ultrasound surveillance for hepatocellular carcinoma is recommended in those with accompanying cirrhosis[7].Treatment with antiviral medication is recommended in all people with proven chronic hepatitis $\mathrm{C}$ who are not risk of dying from other causes[92].People with highest complication risk should be treated first, with the risk of complications based on the degree of liver 
scarring[92]The initial recommended treatment depends on the type of hepatitis $\mathrm{C}$ virus with which a person is infected[92],e.g.,treatment for $\mathrm{HCV}$ genotype Ia,HCV genotype Ib, HCV genotype 2,HCV genotype 3, HCV genotype, $\mathrm{HCV}$ genotype 4, and $\mathrm{HCV}$ genotype 5 or 6 is different[92].

Sofosbuvir with ribavirin and interferon appears to be around $90 \%$ effective in those genotype 1,4,5 or 6 disease[93].Sofosbvir with just ribavirin appears to be 70 to $95 \%$ effective in type 2 and 3 disease but has higher rates of adverse effects[93].Treatment during the first six months is more effective than once hepatitis $\mathrm{C}$ has become chronic[69].

\section{Surgical intervention}

Cirrhosis due to hepatitis $\mathrm{C}$ is a common reason for liver transplantation[69],though the virus usually (80-90\% of cases) recurs afterwards[15].Infection of the graft leads to $10-30 \%$ of people developing cirrhosis within five years[94].Treatment with Pegylated interferon and ribavirin post-transplant decreases the risk of recurrence to $70 \%[95]$.

Several alternative therapies are claimed by their proponents to be helpful for hepatitis $\mathrm{C}$ including milk thistle,ginseng,and colloidal silver[96].However no alternative therapy has shown to improve outcomes in hepatitis $\mathrm{C}$,and no evidence exists that alternative therapies have any effect on the virus at all[96].

\section{Prognosis And Prevention}

The responses to treatment is measured by sustained viral response(SVR),defined as the absence of detectable RNA of the hepatitis $\mathrm{C}$ virus in blood serum for at least 24 weeks after discontinuing the treatment[97], and rapid virological response (RVR) defined as undetectable levels achieved within four weeks of treatment. Successful treatment decreases the future risk of hepatocellular carcinoma by $75 \%$ [98]. Prior to 2012 sustained response occurs in about $40-50 \%$ in people with HCV genotype 1 given 48 weeks of treatment[13].A sustained response is seen in 70-80\% of people with HCV genotyoes 2 and 3 with 24 weeks of treatment[13].A sustained response occurs about $65 \%$ in those with gentotype 4 after 48 weeks of treatment. The evidence for treatment in genotype 6 diseases is sparse and what evidence there is support 48 weeks of treatment at the same doses used for genotype 1 disease [99]

\section{Prevention}

As of 2016,no approved vaccine protects against contracting hepatitis C[100].However, there are a number of vaccines under development and some shown encouraging results[99].A combination of harm reduction strategies, such as the provision of new needles and syringes and treatment of substance use, decreases the risk of hepatitis $\mathrm{C}$ in intravenous drug users by about 75\%[101].Screening of blood donors is important at a national level, as adhering to universal precautions within healthcare facilities[18].In countries where there is an insufficient supply of sterile syringes, medications should be given orally rather than via injection-when possible[35].

\section{Conclusion}

Hepatitis $\mathrm{C}$ related diseases have high mortality worldwide. Disease is more common in Africa and Central and East Asia. Countries with high rates include Egypt, Pakistan and China.High number of deaths due to liver cirrhosis and liver cancer. Diagnosis by blood tests for virus antibodies or RNA.Treatment with antiviral drugs. There is number of vaccines under development.

\section{References}

[1]. Ryan KJ,RayCG,Eds.(2004).Sherris Medical Microbiology(4 ${ }^{\text {th }}$ ed.)McGRaw Hill.pp.551-2.ISBN 0-8385-8529-9.

[2]. Viral Hepatitis:A through E and Beyond. National Institute of Diabetes and Digestive and Kidney Diseases.April 2012.Retrieved 4 February 2016.

[3]. Hepatitis C.World Health Organization(WHO).June 211.Retrieved 2011-07-13.

[4]. Lozano R.Global and regional mortality from 235 causes of death for 20 age groups in 1990 and 2010:a systematic analysis for the Global Burden of Disease Study 2010.Lancet.2012;380(9859):2095-128.

[5]. Hepatitis C Fact Sheet N 164 .WHO July 2015.Retrieved 4 February 2016.

[6]. GBD 2013 Mortality and Causes of Death,Collaborators(17 December 2014)Global, regional, and national age-sex specific allcause and cause-specific mortality for 240 causes of death,1990-2013:a systematic analysis for the Global Burden of Disease Study 2013.Lancet.2013;385(9963):117-71.

[7]. Wilkins T,MalcolmJk,RainaD,etal.Acute hepatitis C .Lancet. 2008;372(9635):321-32.

[8]. Table 4.5.Number and rate of deaths with hepatitis $C$ listed as a cause of death by demographic characteristic and year-United States,2004-2008.Viral Hepatitis on the CDC web site. Centers for Disease Control and Prevention, Atlanta GA.Retrieved 28 July 2013.

[9]. Hepatitis Death Rate Creeps past AIDS.New York Times.27 February 2012.Retrieved 28 July 2013.

[10]. Hepatitis C Kills More Americans than Any Other Infectious Disease.Centers for Disease Control and Prevention. May 4,2016. Retrieved 3 August 2016.

[11]. BlachierM,LeleuH,PeckRM,et al. The burden of liver disease in Europe:a review of available epidemiological data.J Hepatol.2013;58(3):593-608.

[12]. Hepatitis C FAQs for Health Professionals.CDC January 8,2016.Retrieved 4 February 2026. 
[13]. Rosen HR.Clinicalpractice.Chronic hepatitis C infectionEngl J Med.2011;364(25):2429-38.

[14]. FeinstoneSM,KapikianAZ,PurcellRH,etal.Transfusion associated hepatitis not due to viral hepatitis type A or B.N Engl J Med.1975;292:767-770.

[15]. Boyer JL.(2001).Liver Cirrhosis and its development proceeding of the FolkSymposium 115.Springer.pp.344.ISBN 978-0-79238760-2.

[16]. Choo QL,KuoG,WeinerAJ,etal.Isolation of acDNA clone derived from a blood-borne non-A,non-B viral hepatitis genome.Science.1989;244(4902):359-62.

[17]. 2000 Winners Albert Lasker Award for Clinical Medical Research(http://.archieved.org/web/20080225184627/ http://www.laskerfoundation.org/awards/library/2000clinical.shtml) atWaybackMachine(archieved February 25,2008).Retrieved 20 February 2008.

[18]. Ray Stuart C,Thomas David L.HepatitisC.In Mandell ,Gerald L,Bennett,John E DollinRaphel.Mandell Douglas and Benett'sPrinciples and Practice of InfectiousDiseases $\left(7^{\text {th }}\right.$ ed.)Philadelphia,PA:Churchill Living stone.2009.ISBN978-0-443068393.

[19]. Nakano T,LauGM,LauGM,etal.An updated analysis of hepatitis C virus genotypes and subtypes based on the complete coding region.Liver Int.2011;32(2):339-45.

[20]. LeratH,HollingerFB.Hepatitis C virus(HVC) occult infection or occult HCV RNA detection?.J Infect Dis.2004;189(1):3-6.

[21]. ZingnegoAL,GianniniC,GragnaniL,etal.Hepatitis $\mathrm{C}$ virus infection in the immunocompromised host: a complex scanrio with variable clinical impact.JTraditional Med.2012;10(1):158.

[22]. JafariS,CopesR,BahrlouS, etal.Tattooing and the risk of transmission of hepatitis C:a systematic review and meta-analysis(PDF)Int $J$ Infect Dis.2010;14(11):e928.

[23]. SuskindDL,RosenthalP.Chronichepatitis.Adolesc Med Clin.2004;15(1):145-58,x-xi.

[24]. McAllister J,CasionC,DavidsonJ,etal.Long term evolution of the hypervariable regions of hepatitis $\mathrm{C}$ virus in a common sourceinfected cohort.JVirol. 1998; 72:4893-4905.

[25]. Abe KinchauspeG.Transmission of hepatitis C by saliva.Lancet.1991;337:248.

[26]. MeheshwariA,ThuluvathPJ.Mansagement of acute hepatitis C.Clinics in LiverDisease.2016;14(1):169-76.x

[27]. PondeRA.Hidden hazards of HCV transmission.Medical MicrobiologyandImmunnology.2011;200(1):7-11.

[28]. Chronic hepatitis C Virus Advances in Treatment,Promise for the Future.Springer Verlag.2011.p.4.ISBN 978-1-1614-1191-8.

[29]. Xia X,LuoJ,BaiJ,etal.Epidemiology of HCV infection among injection drug users in China:systematic review and metaanalysis.Public health.2008;122(10):990-1003.

[30]. Nelson PK,MathersBM,CowieB,etal.Global epidemiology of hepatitis B and hepatitis C in people who inject drugs results of systematic reviews .Lancet. 2011;378(9791):571-83.

[31]. Imperial JC.Chronic hepatitis hepatitis $C$ in the state prison system:insight into the problems and possible solutions. Expert review of gastroenterology \& hepatology.2010;4(3):355-64.

[32]. Moyer VA,U.S.Preventive Services Task Force..Screening for hepatitis C virus infection in adults:U.S.Preventive Services Task Force recommendation statement.Annals of Inter Med.2013;159(5):349-57.

[33]. Marx John.Rosen'semergencymedicine:concepts and clinical practice $7^{\text {th }}$ edition. Philadelphia, PAA:Mosby/Elsevier.2010.p.1154.ISBN 978-0-323-05472-0.

[34]. Day RA,PaulP,WilliamB,etal.Bunner\&Suddarh's test book of Canadian medical-surgical nursing(Canadian $2^{\text {nd }}$ ed.).Philadelphia,PA:Lippincott William \& Wilkins.2009.p.1237.ISBN 978-7817-9989-8.

[35]. Alter MJ.Epidemiology of hepatitis C virus infection (PDF).World Journal of gastroenterology:WJG.2007;13(17):2436 - 41.

[36]. Highest Rates of Hepatitis C Virus Transmisson Found in Egypt.AI Bawaaba.2010-08-09.Retrieved 2010-08-27.

[37]. TohmeRA,Holmberg SD.Is sexual contact a major mode of hepatitis C virus transmission ?.Hepatology.2010;52(4):1497-505.

[38]. Hepatitis C Group Education Class.United States Department of Veteran Affairs.

[39]. Lock G,Dirscherl M,0bemeier F,etal.Hepatitis C-contamination of toothbrushes: myth or reality ?.J Viral Hepat.2006;13(9):571-3.

[40]. Hepatitis C FAQs for Health Professionals. Centers for Disease Control and Prevention (CDC).Retrieved 2 January 2012.

[41]. Wong T,LeeSS.Hepatitis C:a review for primary care physicians. CMAJ. 2006:174(5):649-59.

[42]. JafariS,CopesR,BaharlouS, etal.Tattooing and the risk of transmission of hepatitis C:a systematic review and meta-analysis(PDF).Int J Infect Dis.2010;14(11):e928-40.

[43]. Hepatitis C(PDF).Centers for Disease Control and Prevention(CDC)Retrieved 2 January 2012.

[44]. Lam NC,GotschPB,LaganRC.Caring for pregnant women and newborns with hepatitis B or C(PDF).American Family Physician.2010;82(10):1225-9.

[45]. Mast EE.Mother- to-infant hepatitis C virus transmission and breastfeeding.AdavanExper Med Biol.2004;554:211-6.

[46]. Dore GJ,KaldorJM,MacCaughanGW.Systematic review of role of polymerase chain reaction in defining infectiousness among people infected with hepatitis C virus.BMJ.1997;315:333-37.

[47]. Thomas DL,VillanoSA,RiesterKA,etal.Perinatal transmission of hepatitis C virus from human immunodeficiency virus type 1infected mothers.J InfectDis. 1998; 177:1480-88.

[48]. EysterME,AlterHJ,AledortLM,etal.Heterosexual co-transmission of hepatitis $\mathrm{C}$ virus(HCV) and human immunodeficiency virus(HIV).Ann Intern Med.1991;115:764-68.

[49]. ThomasDL,ShihJW,AlterHJ,etal.Effect of immunodeficiency virus on hepatitis C virus infection among injecting drug users. $J$ Infect Dis.1996;174:690-95.

[50]. FarciP,AlterHJ,WongD,etal.A long term study of hepatitis C virus replication in non-A,non B hepatitis.NEngl J Med.1991;325:98104.

[51]. SulkowskiMS,RaySC,ThomasDL,etal.Needlestick transmission of hepatitis C.JAMA.2007;287:2406-13.

[52]. VillanoSA,VlahovD,NelsonKE, etal.Persistence of viremia and the importance of long term follow-up after acute hepatitis $C$ infection.Hepatology.1999;29:908-14

[53]. NetskiDM,MosburgerT,DeplaE,etal.Humoral immune response in acute hepatitis C virus infection.Clin Infect Dis.2005;41:667-75.

[54]. Adams G,KuntzS,RabalaisG,etal.Natural recovery from acute hepatitis C virus infection by agammaglobulinemic twin children.Pediatr Infect Dis J.1997;16:533-34.

[55]. ThimmeR,OldachD,ChangKM,etal.Determinants of viral clearance and persistence during acute hepatitis $\mathrm{C}$ virus infectionExp Med.2001;194:395-1406.

[56]. Chang KM,Thimme R, MelpolderJJ,etal.Differential $\mathrm{CD}^{+}$and $\mathrm{CD}^{+} \mathrm{T}$ - cell responsiveness in hepatitis $\mathrm{C}$ virus infection.Hepatology.2001;33:267-76.

[57]. Thomas DL,AstermborskiJ,RaiKM,etal.The natural history of hepatitis $\mathrm{C}$ virus infection:Host,viral and environmental factors.JAMA.2000;284:450-56. 
[58]. Tong MJ,EL-FarraNS,ReikesAR,etal.Clinical outcomes after transfusion associated hepatitis C.N Engl J Med.1995;332:1463-1466.

[59]. HijikataM,ShimizuYK,Katoetal.Equilibrium centrifugation studies of hepatitis $\mathrm{C}$ virus:evidence for circulating immune complexes.J Virol.1993;67:1953-58.

[60]. Choo SH,SoHS,ChoJM,etal.Association of hepatitis C virus particles with immunoglobulin:A mechanism for persistent infection.J Gen Virol.1995;76:2337-2341.

[61]. Miyamato H,Okamoto H,SatoK,etal.Extraordinarily low density of Hepatitis C estimated by sucrose density gradient centrifugation and the polymerase chain reaction.J Gen Virol.1992;73:715-18.

[62]. Cox AI.NetskiDM,MosbrugerT,etal.Prospective evaluation of community acquired acute phase hepatitis $\mathrm{C}$ virus infection.Clin Infect Dis.2005;40:951-58.

[63]. Chronic Hepatitis C virus Advances in Treatment,Promise for the Future.Springer Verlag.2011.pp.103-104.ISBN 978-1-46141191-8.

[64]. FortonDM,AllsopJM,CoxIJ,etal.A review of cognitive impairment and cerebral metabolite abnormalities in patients with hepatitis C infection.AIDS(London, England).2005;19(Suppl):S53-63.

[65]. NicotF(2004).Chapter 19.Liver biopsy in modern medicine.Occult hepatitis C virusinfection:Where are we now ?.ISBN 978-953307-883-0.

[66]. El-ZayadiAR.HepaticSeatosis:a benign disease or silent killer.W Jgasterol:WJG.2008;14(26):4120-6.

[67]. Mueller S,MillonigG,SetizHK.Alcoholic liver disease and hepatitis C: a frequently underestimated combination(PDF) $W J$ gasterol:WJG.2009;15(28):3462-71.

[68]. FattovichG,StroffoliniT,ZangiI,etal.Hepatocarcinoma in cirrhosis:incidence and risk factors.Gasteroenterol.2004;127(5 Suppl 1):S35-50.

[69]. OzarasR,TahanV.Acute hepatitis C:prevention and treatment.Expert view of anti-infective therapy.2009;7(3):351-61.

[70]. Zaltron S, SpinettiA,BiasiL,etal.Chronic HCV infection:epidemiological and clinical relevance.BMC.2012;12Suppl 2:S2.

[71]. DammacoF,SansonnoD.Reviewarticle:Therapy for Hepatitis C virus-Related CryoglobulinemiaVasculitis.NEngl $J$ Med.2013;369(11):1035-1045.

[72]. GignegoAL,FerriC,PileriSA,etal.Extrahepatic manifestations of Hepatitis C Virus infection:a general overview and guidelines for a clinical approach. Digestive andLiver Disease. 2007;39(1):2-17.

[73]. DammacooF,SansonnoD,PiccoliC,etal.The lymphatic system in hepatitis C virus infection autoimmunity. Mixedcryoglulinemia,and Overt B-cell malignancy. Seminarars in liver disease.2000; 20(2):143-57.

[74]. Lee MR,ShumackS,Prunigonodularis:areview.Aust J Dermatol.2005;46(4):211-18.

[75]. MatsumoriA.Role of hepatitis C virus in cardiomyopathies.ErnesrScheringResearch Foundation Workshop.2006;(55):99-120.

[76]. Monaco S,Ferrari S, GajofattoA,etal.HCV- related nervous system disorders..ClinDevelop Immunol.2012; 2012:23618.

[77]. Webster DP,KlenermanP,DusheikoGM.Hepatitis C.Lancet.2015;385(9973):112-1135.

[78]. SugdenPB,CameronB,BullR,etal.Occult infection with hepatitis C virus:friend or foe ?.Immunol Cell Biolog.2012;90(8):763-73.

[79]. CarrenoV.Occult hepatitis C virus infection: a new form of hepatitis C.Wrld JGasterolg.WJG.2006;12(43):6922-5.

[80]. CarreioGV,NebredaJB,AguilarIC,etal.Occult hepatitis C virus infection. Enfermedadesinfecciosasymicronbiologia clinica.2011;29Suppl 3:14-9.

[81]. CarrenioV,BartolomeJ,CastiloI, etal.Occult hepatitis B virus infection and hepatitis C virus infections.Reviews Med Virol.2008;18(3):139-57.

[82]. Arshad M,EI-KamarySS,JhaveriR.Hepatitis C virus infection in pregnancy and newborn period-are they opportunities for treatment.JVirol Hepat.2011;18(4):229-36.

[83]. Hunt CM,CarsonKL,ShararaAI.Hepatitis C in pregnancy.Obstet Gynecol.1997;89(5Pt2):883-90.

[84]. Fischer B.Hepatitis C virus infection.Semin Fetal Neonatal Med.2007;12(3):168-73.

[85]. IndolfiG,RestiM.Perinatal transmission of hepatitis C infection.J Med Virol.2009;81(5):836-43.

[86]. EinazS,KozielMJ.Immuopathogenesis of hepatitis C virus in the immunosuppressed host.Transpl Infect Dis.2002;4(2):85-92.

[87]. Robinson JL.Vertical transmission of the hepatitis C virus.:Current knowledge and issues.Paediatr Child Helath.2008;13(6):529534.

[88]. Moyer VA.Screening for Hepatitis C Virus Infection in Adults:U.S.Preventive Services Task Force Recommendations Statement. Ann Int Med.2013;159(5):349-57.

[89]. Moyer VA.(U.S.Preventive Services Task Force).Screening for hepatitis C virus infection in adults :U.S. Preventive Services Task Force recommendation Statement.AnnInt Med.2013;159(5):349-57.

[90]. Senadhi V.A paradigm shift in the outpatient approach to liver function tests. SouthMed J.2011;104(7):521-5.

[91]. ToressiJ,JohnsonD,WedemyerH.Progress in the development of preventive and therapeutic vaccines for hepatitis C virus(PDF). $J$ Hepatol.2011;54(6):1273-85.

[92]. AASLD/IDSA HCV Guidence,Panel.Hepatitis C guidance:AASLD-IDSA recommendations for testing, managing and treating adults infected with hepatitis C virus. Hepatol.(Baltimore,MD).2015;62(3):932-54.

[93]. De ClereqE.Dancing with chemical formulae of antivirals: A panoramic view (Part 2).Biochem Pharm.2013;86(10):1397-410.

[94]. CiriaR.PleguezueloM,KhorsandiSE,etal.Strategies to reduce hepatirtis C virus recurrence afterlivertransplantation.World $J$ Hepatol.2013;5(5):237-50.

[95]. CoillyA,RocheB,SamuelD.Current management and perspectives for HCV recurrence after liver transplantation.Liver Int.2013;33Suppl 1:56-62.

[96]. Hepatitis C and CAM:What Science Says (Http:// nccam.nih. gov/ health/ providers/digest/hepatitis C-science.htm)National Center for Complementary and Alternative Medicine(NCCAM).March 2011.(retrieved 7 March 2011).

[97]. Helms RA,Quan David J.,eds.Textbook of Therapeutics:Drug and DiseaseManagement( ${ }^{\text {th }}$ Ed.)Philadelphia.Pa.(u.a)Lippincott Williams \& Wilkins.p1340. ISBN0 -7818-5734-7.Retrieved 7 Novemver 2014.

[98]. Morgan RL.BaackB,SmithBD,etal.Eradication of Hepatitis C virus Infection and the development of Hepatocellular Carcinoma: A Meta-analysis of the OservationalStudies.Annals of Int Med.2013;158(5 Pt 1):329-37.

[99]. Fung J,LaiCL,HuangI,etal.Chronic hepatitis C virus genotype 6 infection: response to pegylated interferon and ribavirin.J Infect Dis.2008;198(6):808-12.

[100]. AbdelwahabKS,Ahmed Said ZN.Status of hepatitis C virus vaccination.Recentupdate. World J Gastroenterol.2016;22(2):862-73.

[101]. Hagan H,PougetER,DesJarlais DC.A systematic review and meta-analysis of interventions to prevent hepatitis C virus infection in people who inject drugs. JInfect Dis.2011;204(1):74-83. 